\title{
44-GHz class I methanol maser survey towards 6.7-GHz class II methanol masers
}

\author{
Do-Young Byun, Kee-Tae Kim and Jae-Han Bae \\ Korea Astronomy and Space Science Institute, \\ 776, Daedeokdae-ro, Yuseong-gu, Daejeon, Republic of Korea \\ email: bdy@kasi.re.kr
}

\begin{abstract}
The Class II 6.7-GHz methanol maser is a tracer of high mass young stellar objects. We present results of a $44-\mathrm{GHz}$ class I methanol maser and $22-\mathrm{GHz}$ water maser survey using the KVN (Korean VLBI Network) 21-m single dish radio telescopes towards 284 6.7-GHz maser sites. Class I methanol maser and water maser emission is detected towards 116 (41\%) and 136 (48\%) sources, respectively. About 50 sources have a peak flux density higher than $10 \mathrm{Jy}$ at 44-GHz. They are candidates for VLBI studies using the KVN.
\end{abstract}

Keywords. masers, surveys, stars: formation, stars: outflows

\section{Introduction}

Since high mass young stellar objects (HMYSOs) are deeply embeded in natal molecular clouds and their distances are usually large, it is hard to study their environment in detail. Consequently, the physical processes of HMYSOs, such as jet formation, mass infall and disk formation, are not yet fully understood. Class I methanol maser emission is excited in the interface region between the outflow of a central object and the surrounding molecular material (Kurtz et al. 2004 etc.). This methanol maser line is therefore a very useful tool to study the outflows of HMYSOs.

We present the results of a $44-\mathrm{GHz}$ class I methanol maser and $22-\mathrm{GHz}$ water maser survey towards $6.7-\mathrm{GHz}$ methanol sites. The $6.7-\mathrm{GHz}$ class II methanol maser is known to occur only near HMYSOs. The primary aim of this study is to build a data base of 44-GHz methanol masers for future VLBI studies of HMYSOs using the KVN. Additionally, a statistical analysis using the survey results is used to study the relation between maser occurence and the physical condition of HMYSOs.

\section{Observations and Results}

We selected 284 targets with a declination higher than -30 degree among 519 sources in the catalog of $6.7-\mathrm{GHz}$ methanol masers of Pestalozzi et al. (2005). 44-GHz methanol maser observations towards the selected targets were made with the KVN 21-m radio telescopes at Yonsei and Ulsan during October and November 2009. The on-source integration time of 20 or 30 minutes on each source resulted in a typical rms noise level of $0.7 \mathrm{Jy}$ at $0.2 \mathrm{~km} \mathrm{~s}^{-1}$ velocity resolution. The $22-\mathrm{GHz}$ water maser observations towards the same targets were made during May and June 2010. The rms noise levels of the water maser spectra are $\sim 1.2 \mathrm{Jy}$. The beam size of the $21-\mathrm{m}$ telescope at the $44-\mathrm{GHz}$ and $22-\mathrm{GHz}$ is $65^{\prime \prime}$ and $125^{\prime \prime}$, respectively (Lee et al. 2011).

The 44-GHz methanol masers and water masers are detected towards 116 and 136 of 284 targets equating to detection rates of $41 \%$ and $48 \%$, respectively. Fig. 1 shows that more than 50 sources have a peak flux density at 44-GHz methanol stronger than $10 \mathrm{Jy}$, 


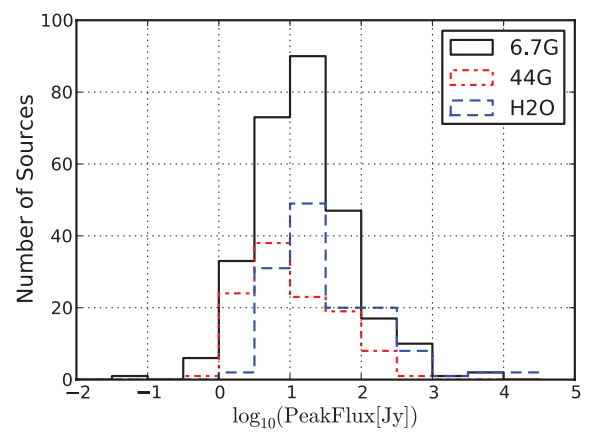

Figure 1. Peak flux density distribution.

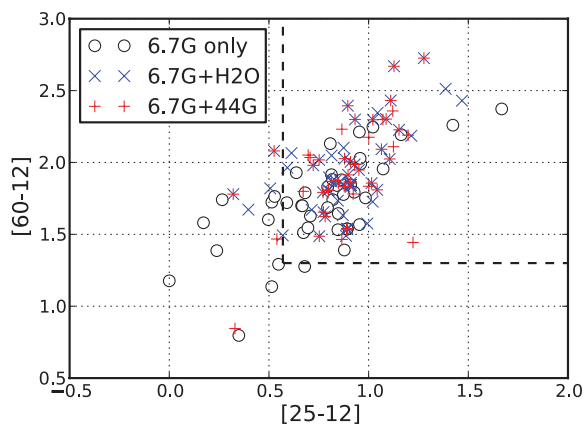

Figure 3. IRAS color-color plot.

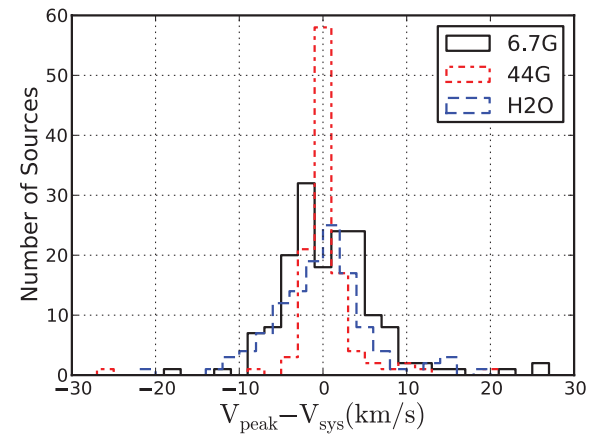

Figure 2. Peak velocity distribution.

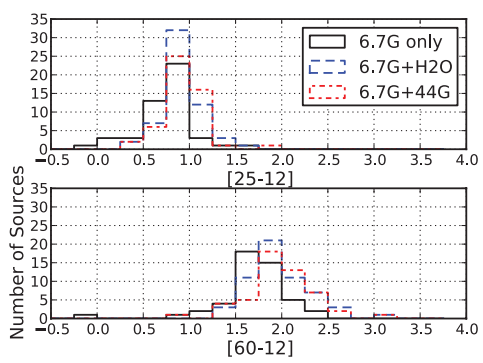

Figure 4. IRAS color distribution.

which is around the 7- $\sigma$ detection level of the KVN baseline. We find that $3 / 4$ of the class I methanol maser sources are associated with water masers. This implies that class I methanol masers and water masers share a common evolutionary stage. We compared the peak velocities of the maser emission with the systematic velocities of HMYSOs determined from thermal molecular lines tracing dense regions. Fig. 2 shows that the peak velocities of $44-\mathrm{GHz}$ methanol masers are concentrated around the systematic velocities more tightly than the other masers.

We investigated the maser occurence rates with the IRAS colors. For this investigation, 116 IRAS associated sources with reliable colors were selected. Figs. 3 and 4 show that the maser occurence rates of $44-\mathrm{GHz}$ methanol and $22-\mathrm{GHz}$ water masers increase systematically with IRAS colors. This seems to imply that 44-GHz methanol maser and 22-GHz water maser emission increases with luminosity as the central object evolves from protostar to UCHII, considering the UCHII have larger IRAS colors.

It should be noted, however, that the IRAS association could be mistaken due to the limited positional accuracies of the single dish observations of masers and the IRAS data, since massive star forming regions often contain multiple HMYSOs within a few arcsec scale. This can contaminate the maser occurence rates with IRAS colors. Study with higher angular resolution is neccessary to confirm the relation between the maser occurence and the evolutionary stage of HMYSO.

\section{References}

Kurtz, S., Hofner, P., \& Álvarez, C. V. 2004, ApJS, 155, 149

Lee, S. S. et al. 2011, PASP, 123, 1398

Pestalozzi, M., Minier, V., \& Booth, R. S. 2005, A\&A., 432, 737 